\section{ELECTRON CYCLOTRON RADIATIVE TRANSFER IN THE PRESENCE OF POLARIZATION SCRAMBLING IN WALL REFLECTIONS}

\section{F. Albajar ${ }^{1}$, M. Bornatici ${ }^{2}, F$. Engelmann ${ }^{3}$}

${ }^{1}$ Departament de Física i Enginyeria Nuclear, Universitat Politècnica de Catalunya, Barcelona, Spain

${ }^{2}$ INFM, Dipartimento di Fisica "A.Volta", Università di Pavia, Pavia, Italy ${ }^{3}$ Max-Planck-Institut für Plasmaphysik, Garching, Germany

$$
\text { e-mail: bornatici@fisicavolta.unipv.it }
$$

An exact analytical solution of the equation of radiative transfer is obtained for a cylindrical system with specularly reflecting walls, accounting for the presence of polarization scrambling in the reflection process. The effects of polarization scrambling on the specific intensity of the radiation can be described via an effective wall reflection coefficient for the extraordinary $(x)$ and ordinary $(o)$ mode. For the special case of electron cyclotron radiation in a fusion plasma, a numerical analysis of the impact of polarization scrambling on both the specific intensity of the radiation and the radial profile of the net power radiated as well as on the total power loss is carried out for ITER-like parameters in steady-state operation.

\section{Introduction}

It is known for some time that the mixing of the polarizations of the extraordinary $(x)$ and ordinary $(o)$ mode that occurs in the wall reflection process, to be referred to as "polarization scrambling", has to be accounted for in the diagnostics based on electron cyclotron (EC) emission [1,2,3]. The effect of polarization scrambling has also been considered in the context of EC plasma heating [4]. Less attention has been paid to the impact that such an effect might have on the EC radiative transfer in fusion plasmas, the only exceptions being the CYTRAN routine developed by Tamor [5] and a formal approach in an analysis of passive EC current generation [6].

Here the impact of polarization scrambling on both the specific intensity of the EC radiation and the radial profile of the net EC radiative power density as well as on the total power loss in fusion plasmas is investigated on the basis of a special analytical solution of the equation of radiative transfer.

\section{Solution of the radiative transfer equation (RTE) with polarization scrambling}

On referring to a radiating system with (circularly) cylindrical geometry and specularly reflecting walls, the (exact) solution of the RTE for the specific inten- sity of the radiation in the mode $\sigma=x, o$ at the generic point $s=s(\mathbf{r})$ of the ray in the direction $\hat{\mathbf{s}}, I^{\sigma}(s, \omega, \hat{\mathbf{s}}) \equiv I^{\sigma}(s)$, is (cf. [7])

$$
\frac{I^{\sigma}(s)}{\left(N_{r}^{\sigma}(s)\right)^{2}}=i^{\sigma}\left(s, s_{0}\right)+I_{r e f}^{\sigma}\left(s_{0}\right) \mathrm{e}^{-\tau^{\sigma}\left(s, s_{0}\right)}
$$

where $N_{r}^{\sigma}$ is the refractive index,

$$
i^{\sigma}\left(s, s_{0}\right)=\int_{s_{0}}^{s} d s^{\prime} \frac{j^{\sigma}\left(s^{\prime}\right)}{\left(N_{r}^{\sigma}\left(s^{\prime}\right)\right)^{2}} \mathrm{e}^{-\tau^{\sigma}\left(s, s^{\prime}\right)}=\frac{\omega^{3}}{8 \pi^{3} c^{2}} \int_{s_{0}}^{s} d s^{\prime} T\left(s^{\prime}\right) \alpha^{\sigma}\left(s^{\prime}\right) \mathrm{e}^{-\tau^{\sigma}\left(s, s^{\prime}\right)},
$$

with $j^{\sigma}$ and $\alpha^{\sigma}$, respectively, the emission and absorption coefficients and $\tau^{\sigma}\left(s, s^{\prime}\right) \equiv \int_{s^{\prime}}^{s} d s^{\prime \prime} \alpha^{\sigma}\left(s^{\prime \prime}\right)$ the optical thickness relative to the ray path between the two arbitrary points $\left(s_{0} \leq\right) s^{\prime}$ and $s$. The 2 nd form of Eq. (2) refers to the case of local thermodynamic equilibrium at (local) temperature $T(s(\mathbf{r}))$. The 2 nd term on the r.h.s. of Eq. (1) has to do with the wall reflection process at the generic point $s_{0}$, for which one has, in the presence of polarization scrambling,

$$
I_{\text {ref }}^{\sigma}\left(s_{0}\right)=\frac{1}{1-R_{e f f}^{\sigma \sigma^{\prime}} e^{-\tau^{\sigma}}}\left[R_{e f f}^{\sigma \sigma^{\prime}} i^{\sigma}+\frac{R_{w}^{\sigma^{\prime}} p^{\sigma^{\prime}}}{R_{w}^{\sigma} p^{\sigma}} \frac{R_{w}^{\sigma}-R_{e f f}^{\sigma \sigma^{\prime}}}{1-R_{w}^{\sigma^{\prime}} e^{-\tau^{\sigma^{\prime}}}} i^{\sigma^{\prime}}\right], \quad \sigma \neq \sigma^{\prime} .
$$

In Eq. (3),

$$
R_{e f f}^{\sigma \sigma^{\prime}}=R_{w}^{\sigma}\left[1-p^{\sigma} \frac{1-R_{w}^{\sigma^{\prime}} e^{-\tau^{\sigma^{\prime}}}}{1-R_{w}^{\sigma^{\prime}}\left(1-p^{\sigma^{\prime}}\right) e^{-\tau^{\sigma^{\prime}}}}\right],
$$

to be referred as the effective wall reflection coefficient, with $R_{w}^{\sigma}(\leq 1)$ the wall reflection coefficient of the radiation in the mode $\sigma[8]$ and $p^{\sigma}(\leq 1)$ the polarization scrambling parameter which quantifies the percentage of radiation transferred from the mode $\sigma$ to the mode $\sigma^{\prime}$ in each (specular) reflection. Furthermore, $\tau^{\sigma} \equiv \tau^{\sigma}\left(s_{0}, s_{1}\right)$ and $i^{\sigma} \equiv i^{\sigma}\left(s_{0}, s_{1}\right)$, with $s_{0}$ and $s_{1}$ a pair of consecutive reflection points. The result (3)-(4) is obtained on solving the RTE with the boundary conditions shown in Fig. 1 and proceeding along the same lines as in the absence of polarization scrambling [7]

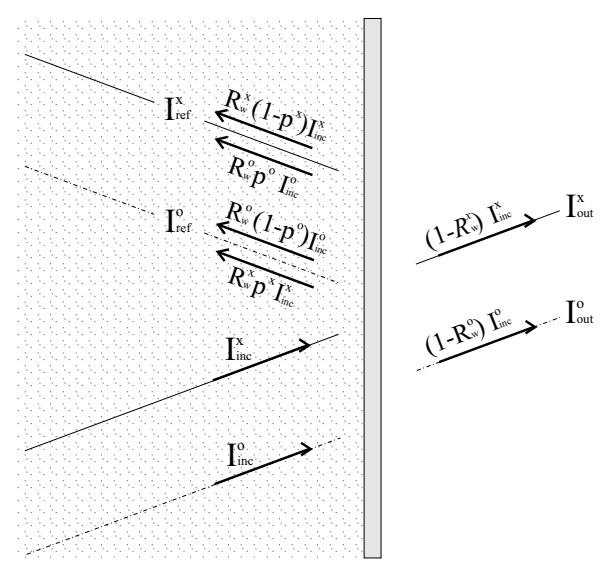

Figure 1: Schematic diagram of the boundary conditions used to solve the radiative transfer equation for the case of mode-dependent reflection and polarization scrambling. The subscripts "inc" (incoming), "ref" (reflected) and "out" (outgoing) refer, respectively, to the specific intensity of the radiation at the generic point $s_{0}$ where the ray hits the wall. 
The effective wall reflection coefficient (4) accounts for the net loss of power from the mode $\sigma$ to the mode $\sigma^{\prime}$ during wall reflection through polarization scrambling, also depending on re-absorption; $R_{e f f}^{\sigma \sigma^{\prime}} \leq R_{w}^{\sigma}$, i.e., the polarization scrambling reduces the genuine wall reflection, this effect being the stronger the larger is the optical thickness $\tau^{\sigma^{\prime}}$. Note also that $R_{\text {eff }}^{\sigma \sigma^{\prime}}\left(p^{\sigma^{\prime}}=0\right)=R_{w}^{\sigma}\left(1-p^{\sigma}\right)$, that is equal to the expression obtained in the optically thick limit $\tau^{\sigma^{\prime}} \gg 1$.

Polarization scrambling, in the strict sense of the concept, is described by the 2nd term on the r.h.s of Eq. (3). Whereas for the $x$-mode (interacting strongly with the plasma) the 1 st term $\left(\sim i^{x}\right)$ on the r.h.s. of Eq. (3) is larger than the 2 nd one notwithstanding the strength of polarization scrambling, i.e., $I_{r e f}^{x}\left(s_{0}\right) \simeq\left[R_{e f f}^{x o} /\left(1-R_{e f f}^{x o} e^{-\tau^{x}}\right)\right] i^{x}$, with the result that the specific intensity $I^{x}(s)$, cf. Eq. (1), is a (weakly) decreasing function of $p^{x}$, for the (weakly interacting) $o$ mode the polarization scrambling tends to make the 2 nd term $\left(\sim i^{x}\right)$ of Eq. (3) the dominant one, already for small values of $p^{x}$; for $p^{x} \gtrsim 0.5$, the 2 nd term alone is an excellent approximation:

$$
I_{r e f}^{o}\left(s_{0}\right) \simeq \frac{R_{w}^{x} p^{x}}{R_{w}^{o} p^{o}} \frac{R_{w}^{o}-R_{e f f}^{o x}}{\left(1-R_{e f f}^{o x} e^{-\tau^{o}}\right)\left(1-R_{w}^{x} e^{-\tau^{x}}\right)} i^{x}=\frac{R_{w}^{x} p^{x}}{\left(1-R_{e f f}^{o x} e^{-\tau^{o}}\right)\left[1-R_{w}^{x}\left(1-p^{x}\right) e^{-\tau^{x}}\right]} i^{x},
$$

Eq. (5) expressing the fact that the $o$-mode then is mainly generated from the $x$ mode through polarization scrambling. Generally $I^{o}(s)$ does increase with increasing $p^{x}$. The numerical evaluation of Eq. (1) confirms the significant enhancement (weak decrease) of the specific intensity of the ordinary (extraordinary) mode due to polarization scrambling, as shown in Fig. 2 for the case for which both the wall reflection coefficients and polarization scrambling are mode independent, i.e., $R_{w}^{x}=R_{w}^{o}(=0.6)$ and $p^{x}=p^{o}$.

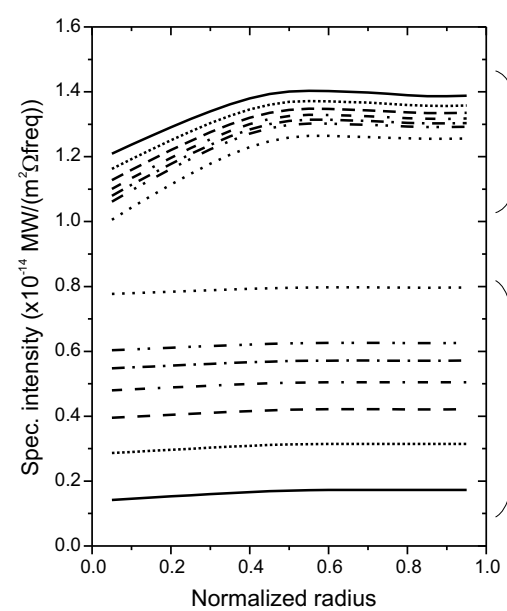

Figure 2: Extraordinary and ordinary radial profiles of the specific intensities at the frequency $\omega=8 \omega_{c}$ for a ray crossing the centre of the plasma in a cross-section perpendicular to the magnetic field for a wall reflection coefficient $R_{w}=0.6$ and various values of the polarization scrambling parameter $p$. An ITER-like fusion plasma is considered with parameters such that $R=6.2 \mathrm{~m}, a=2.0 \mathrm{~m}$, $B_{t}=5.3 \mathrm{~T}, \quad n_{e}=n_{e 0}\left(1-\rho^{2}\right)^{\text {rn }} \quad$ with $n_{e 0}=1.1 \times 10^{20} \mathrm{~m}^{-3}$ and $\gamma_{\mathrm{n}}=0.1$, and $T_{e}=T_{e 0}\left(1-\rho^{\beta \mathrm{T}}\right)^{\gamma \mathrm{T}}$ with $T_{e 0}=45$ $\mathrm{keV}, \beta_{\mathrm{T}}=5.4$ and $\gamma_{\mathrm{T}}=8$.

\section{The effect of polarization scrambling \\ on the EC radiative power density}

To obtain a quantification for the upper limit of polarization scrambling effects on the net EC radiative power density [7],

$\frac{d P_{E C}(\mathbf{r})}{d V}\left(=\sum_{\sigma=x, o} \frac{d P_{E C}^{\sigma}(\mathbf{r})}{d V}\right)=\sum_{\sigma=x, o} \int_{\omega_{\min }^{\sigma}}^{\infty} d \omega \int d^{2} \Omega_{\hat{\mathbf{s}}} \alpha^{\sigma}(\mathbf{r}, \omega, \hat{\mathbf{s}})\left[I_{b b}^{\sigma}(\mathbf{r}, \omega)-I^{\sigma}(\mathbf{r}, \omega, \hat{\mathbf{s}})\right],(6)$

where $I_{b b}^{\sigma} \equiv\left(N_{r}^{\sigma}\right)^{2} \omega^{2} T(\mathbf{r}) / 8 \pi^{3} c^{2}$ is the blackbody intensity, has been evaluated numerically from Eqs. (1)-(3) comparing the extreme case $p=1$ with that of no scrambling. A sample of the numerical results is shown in Figs. 3 and 4 for the wall reflection coefficients $R_{w}=0.8$ and $R_{w}=0.6$, respectively. The quantity

$$
\Delta^{\sigma}(p) \equiv \frac{\left|d P_{E C}^{\sigma} / d V(p)-d P_{E C}^{\sigma} / d V(p=0)\right|}{d P_{E C}^{\sigma} / d V(p=0)}
$$

is a measure for the relative importance of polarization scrambling. From these figures it appears that (i) the polarization scrambling tends to increase (diminish) the contribution to the net EC radiative power density from the extraordinary (ordinary) mode. This is in agreement with the (opposite) trend of the corresponding specific intensities, cf. Fig. 2, on noting that it is the difference $\left(I_{b b}-I^{\sigma}\right)$ that is relevant to $d P_{E C}^{\sigma} / d V$, cf. Eq. (6). For the $x$-mode, the enhancement is close to $20 \%$ for $R_{w}=0.8$, cf. Fig. 3 , and about $12 \%$ for $R_{w}=0.6$, cf. Fig. 4 , in the inner half of the plasma cross-section, and is larger than the decrease occurring for the $o$-mode. This result has to do with the average inherent in the integration over the solid angle in Eq. (6), which tends, in particular, to reduce the significant enhancement of the specific intensity of the $o$-mode due to polarization scrambling found for the central ray, cf. Fig. 2; (ii) the total (summed over $x$ and $o$ modes) net EC radiative power density is increased by polarization scrambling, by about $9 \%$ in the central plasma region, as shown in Fig. 5 . The dependence of the total EC power loss on polarization scrambling is also given in Fig. 5.

In conclusion, the effect of polarization scrambling in a fusion plasma is found to significantly enhance the specific intensity of the radiation of the ordinary mode at the expense of a weakening of the corresponding intensity of the extraordinary mode for rays propagating at large angles with respect to the magnetic field. Whether this fact has an important impact on the passive EC current generation in a fusion plasma [6] needs to be assessed. On the other hand, polarization scrambling turns out to influence only weakly the net EC radiative power density and the total EC power loss. 


\section{References}

[1] A. E. Costley et al., Phys. Rev. Lett., 1974, 33, 758

[2] I. H. Hutchinson and D. S. Komm, Nucl. Fusion, 1977, 17, 1077.

[3] R. M. J. Sillen et al., Nucl. Fusion, 1986, 26, 303.

[4] S. Nowak et al., Fusion Engineering and Design, 2001, 53, 315.

[5] S. Tamor, "A Simple Fast Routine for Computation of Energy Transport by Synchrotron Radiation in Tokamaks and Similar Geometries", Rep. SAI-023-81-189LJ/LAPS-72 (La Jolla, CA: Science Applications, 1981).

[6] W. Kernbichler and S. V. Kasilov, Phys. Plasmas, 1996, 3, 4128

[7] F. Albajar, M. Bornatici, and F. Engelmann, Nucl. Fusion, 2002, 42, 670

[8] R. A. Krajcik, Nucl. Fusion, 1973, 13,
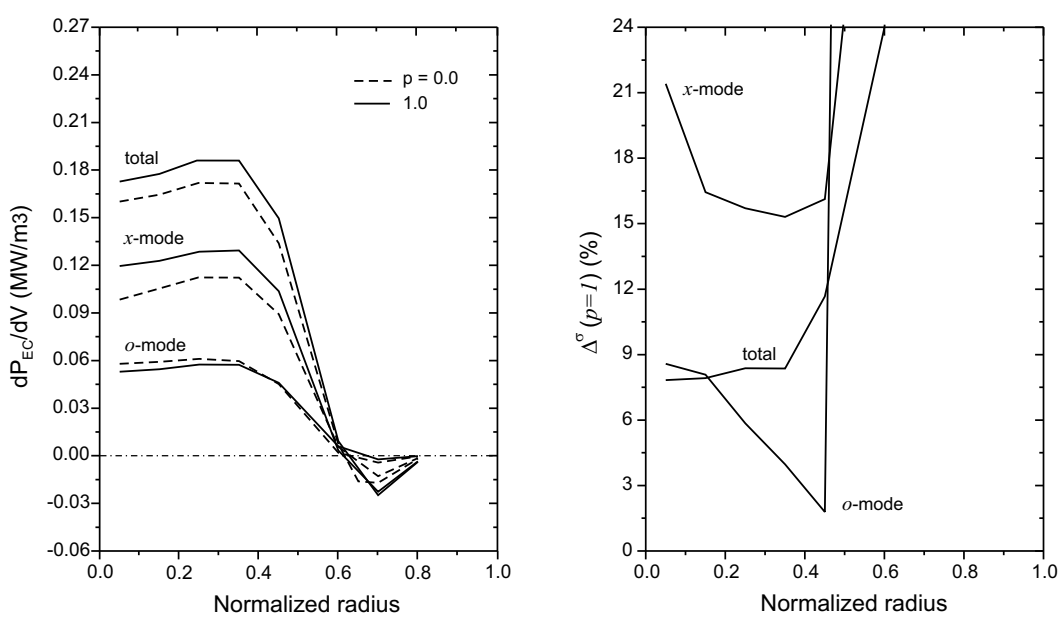

Figure 3: On the left-hand side, the radial profile of the net EC radiative power density for both the single ( $x$ and $o$ ) mode and the total (summed over the two modes), for a wall reflection coefficient $R_{w}=0.8$ is given. The full and dashed curves refer, respectively, to polarization scrambling, $p=1$ and $p=0$. The relative importance of polarization scrambling, for $p=1$, is shown on the right-hand side.
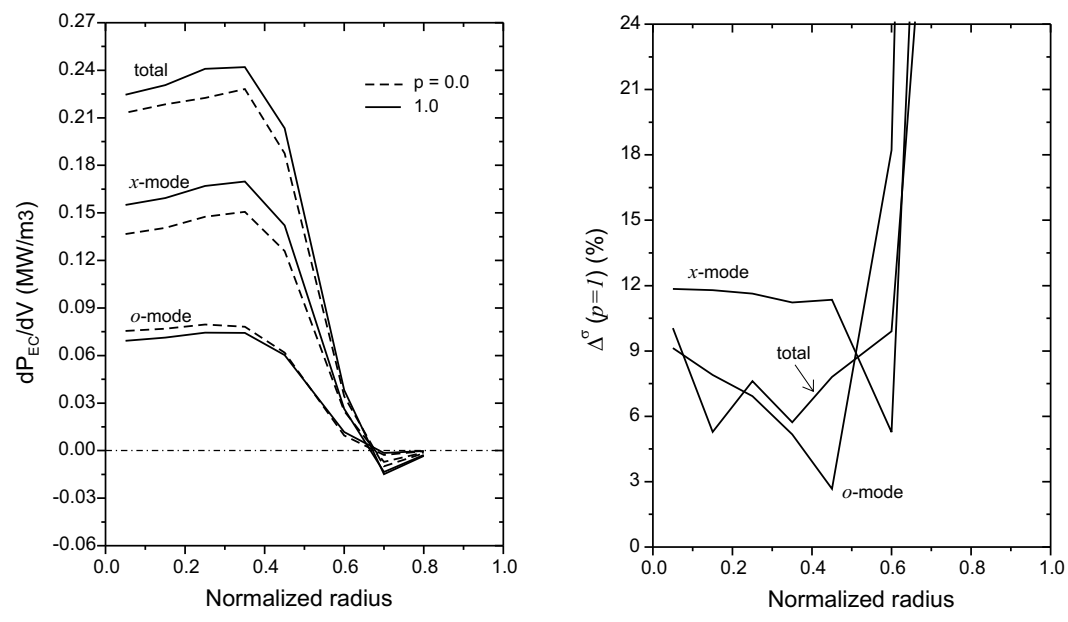

Figure 4: The same as Fig. 3, for a wall reflection coefficient $R_{w}=0.6$
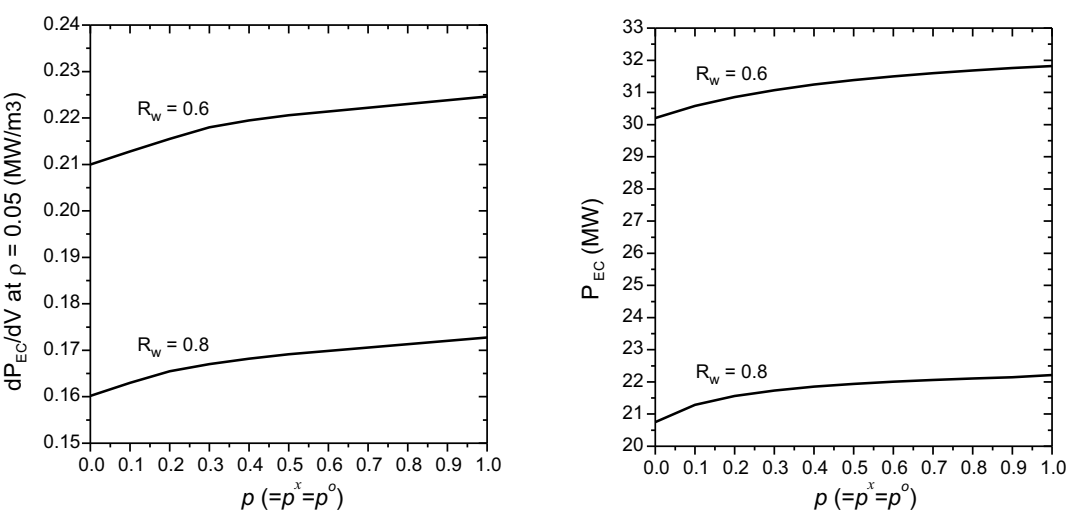

Figure 5: Dependence of the central net EC radiative power loss (left) and of the total EC power loss (right) on the value of $p$ for $R_{w}=0.8$ and 0.6. The former dependence is fitted by $d P_{E C} / d V=d P_{E C} /\left.d V\right|_{p=0}+C_{1} p^{x_{p}}$ with $x_{p}$ about 0.4-0.6. 